\section{Periostin, a matrix protein, is a novel biomarker for idiopathic interstitial pneumonias}

\author{
M. Okamoto*, T. Hoshino*, Y. Kitasato*, Y. Sakazaki*, T. Kawayama*, K. Fujimoto\#, \\ K. Ohshima", H. Shiraishi ${ }^{+}$, M. Uchida ${ }^{+}$, J. Ono ${ }^{\S}$, S. Ohta ${ }^{f}$, S. Kato**, \\ K. Izuhara ${ }^{+, f}$ and H. Aizawa* ${ }^{\dagger}$
}

ABSTRACT: Idiopathic interstitial pneumonias (IIPs) are histopathologically classified into several types, including usual interstitial pneumonia (UIP), nonspecific interstitial pneumonia (NSIP) and cryptogenic organising pneumonia (COP). We investigated whether periostin, a matrix protein, could be used as a biomarker to assess histopathological types of IIPs.

We performed immunohistochemical analyses in each histopathological type of IIP, examined serum levels of periostin in IIP patients and analysed the relationship between serum levels of periostin and the pulmonary functions in patients with idiopathic pulmonary fibrosis (IPF).

Periostin was strongly expressed in lungs of UIP and fibrotic NSIP patients, whereas expression of periostin was weak in the lungs of cellular NSIP and COP patients, as well as in normal lungs. Serum levels of periostin in IPF were significantly higher than those of healthy subjects and COP patients. Furthermore, periostin levels in IPF patients were inversely correlated with their pulmonary functions.

Thus, we have found that periostin is a novel component of fibrosis in IIP. Periostin may be a potential biomarker to distinguish IIP with fibrosis.

KEYWORDS: Biomarker, idiopathic pulmonary fibrosis, lung fibrosis, matrix protein, usual interstitial pneumonia

I diopathic interstitial pneumonias (IIPs) are acute or chronic lung disorders of unknown aetiology characterised by variable degrees of fibrosis and interstitial or intra-alveolar inflammation [1-4]. Based on their histopathological features, IIPs are classified into several types including usual interstitial pneumonia (UIP), nonspecific interstitial pneumonia (NSIP) and cryptogenic organising pneumonia (COP). These three major types account for $\sim 80 \%$ of IIPs, among which UIP, clinically diagnosed as idiopathic pulmonary fibrosis (IPF), is the most common, whereas the rest occur less frequently. IPF/UIP shows fibrosis-dominant features constituted by overproduction and disorganised deposition of extracellular matrix (ECM) proteins, including type I, III and IV collagens, fibronectin and tenascin- $C$, together with abnormal proliferation of mesenchymal cells, distortion of pulmonary architecture and generation of subepithelial fibrotic foci (FF) $[5,6]$. In contrast, COP, characterised by excessive proliferation of granulation tissue within the alveolar ducts leading to chronic inflammation in the surrounding alveoli, rarely shows severe fibrotic changes in the lung. NSIP, defined as a temporally uniform interstitial pneumonia, can be divided into cellular NSIP (cNSIP) and fibrotic NSIP (fNSIP). cNSIP, further divided into group I or group II depending on the absence or presence of concomitant lung fibrosis, respectively, has prominent inflammatory components and severe lung fibrotic changes are uncommon in both cNSIP and COP, whereas fibrosis is predominant in fNSIP (group III) [3, 4].

It is well known that the prognoses and the responses to corticosteroid treatment by the IIP patients can be attributed to histopathological types. IPF/UIP has a grave prognosis with 5-yr mortality rates of $\sim 60-80 \%[3,4]$. In contrast, cNSIP and COP have better prognoses (5-yr mortality rates are $<10 \%$ in cNSIP and $<5 \%$ in COP, and deaths are rare in COP). Most cNSIP and COP patients show a good response to corticosteroids. Mortality rates for fNSIP are intermediate between IPF/UIP and cNSIP or COP, ranging widely from $11 \%$ to $68 \%$ in various studies. The poor prognoses of IPF/UIP and
AFFILIATIONS

Depts of *Medicine,

${ }^{\text {\#Radiology, }}$

"Pathology, Division of Respirology, Neurology, and Rheumatology, Kurume University School of Medicine, Kurume,

Depts of ${ }^{+}$Biomolecular Sciences, fLaboratory Medicine, Division of Medical Biochemistry, Saga Medical School, Saga,

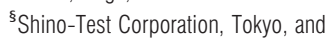
**Division of Pathology and Cell Biology, Graduate School and Faculty of Medicine, University of the Ryukyus, Okinawa, Japan.

CORRESPONDENCE T. Hoshino

Division of Respirology, Neurology, and Rheumatology

Kurume University School of

Medicine

Kurume

Fukuoka 830-0011

Japan

E-mail: hoshino@

med.kurume-u.ac.jp

Received:

April 182010

Accepted after revision:

Dec 072010

First published online:

Dec 222010

European Respiratory Journal Print ISSN 0903-1936 Online ISSN 1399-3003 
fNSIP are thought to be due to resistance to corticosteroids and to impairment of pulmonary functions by fibrosis [3, 4]. Thus, it is important to diagnose the histopathological types of IIP to predict prognoses and responses to corticosteroid treatment. However, to date, no good biomarker has been available for this purpose.

Periostin is a $90-\mathrm{kD}$ ECM protein, composed of an EMI domain in its N-terminal portion, four tandemly lined fasciclin I domains in the middle, and an alternative splicing domain in its C-terminal portion [7, 8]. Periostin plays an important role in the maintenance and development of bones, teeth and the heart, and contributes to tumour progression in several tumour cells. A lot of evidence has recently accumulated showing that expression of periostin is involved in various pathophysiological statuses of fibrosis, including the healing process in myocardial infarction [7] and bone marrow fibrosis [9]. Furthermore, we have recently found that periostin contributes to the formation of fibrosis in response to interleukin (IL)- 4 or IL-13 [10, 11]. However, it remains unknown as to whether periostin is involved in the pathogenesis of IIP.

We examined whether periostin is expressed in lung tissues in IIPs and whether serum levels of periostin can be detected in IIP patients reflecting expression of periostin in the lung tissues. In addition, the possible role of periostin in the pathogenesis of lung fibrosis will be discussed.

\section{MATERIALS AND METHODS Study subjects}

Characteristics of IIP patients enrolled in this study are shown in table 1. 92 patients $(63$ males and 29 females aged $63.5 \pm 1.1 \mathrm{yrs})$ were diagnosed as having IIPs including 51 with IPF, 20 with fNSIP, seven with cNSIP and 14 with COP. All IIP patients had been consecutively monitored from 1994 to 2008 at the Kurume University Hospital (Kurume, Japan), the National Kyushu Medical Center (Fukuoka, Japan), the National Omuta Hospital (Omuta, Japan), the Yame General Hospital (Yame, Japan) and/or the Japan Social Insurance Tagawa Hospital (Tagawa, Japan). All patients were also monitored by their primary care physicians. All IPF patients were diagnosed by at least two respirologists in accordance with the clinical criteria established by the American Thoracic Society/European Respiratory Society, as previously reported [2]. 28 IPF patients were histopathologically diagnosed as UIP by surgical lung biopsy (SLB). All 20 fNSIP and seven cNSIP patients were histopathologically diagnosed by SLB. Out of 14 COP patients, five were histopathologically diagnosed by SLB and nine by transbronchial lung biopsy. The essential pathological feature of COP was the presence of granulation tissue (Masson bodies) and inflammatory cells in the interstitium with preservation of the alveolar architecture [12]. All histopathological diagnoses were performed by at least two professional pathologists. Other diseases such as connective tissue diseases, infection and hypersensitivity pneumonia were excluded. Clinical features, chest high-resolution computed tomography (HRCT) and bronchoalveolar lavage fluid (BALF) cell counts were consistent with COP. In addition, all of the patients showed improvement with or without corticosteroid treatment. All patients had been clinically stable with no disease exacerbations for $\geqslant 3$ months prior to this diagnosis. Diagnosis for acute exacerbation (AE) of IPF was defined in accordance with the criteria detailed in a previous study [13]. Eight IPF patients showed AE. Importantly, arterial oxygen pressure was lower by $>10$ Torr in all of the patients. We have analysed serum levels of procalcitonin, $\beta$-D glucan and cytomegalovirus antigen, and we also examined bacteria and mycobacteria in the sputa of patients to exclude infectious diseases as a cause of the symptoms. However, no infections were observed in the patients. We could not perform bronchoalveolar lavage as we could not obtain written informed consent. We obtained serum samples from most patients at the time of diagnosis and also from age-matched healthy volunteers who served as controls. Control normal lung tissues were obtained from 13 nonsmokers who underwent lung cancer extirpation after surgery at Kurume University Hospital. Sample collection and all procedures were approved by the ethics committees of Kurume University in accordance with the ethical standards of the Helsinki Declaration of 1975. Informed consent was obtained from all patients and healthy volunteers.

\section{HRCT review}

Briefly, patients were classified as having a definite UIP pattern when the HRCT demonstrated honeycombing with predominantly peripheral and basal distribution. Patients were classified as having a probable UIP pattern when the HRCT demonstrated a reticular pattern with predominantly peripheral and basal distribution but only minimal size or with no honeycombing. In this study, two expert chest radiologists independently evaluated the HRCT of all patients. In the case of disagreement, the radiologists re-examined the scans in question to reach a consensus. The radiologists were blinded to patient identification, treatment assignment and temporal sequence of the studies, as in a previous study [13].

\section{BALF}

BALF was obtained by washing the right middle lobe or left lingular segment three times with $50 \mathrm{~mL}$ of physiological saline (total $150 \mathrm{~mL}$ ).

\section{Pulmonary function test}

Pulmonary function tests including vital capacity (VC), forced vital capacity (FVC) and forced expiratory volume in $1 \mathrm{~s}$ were performed using an electrical spirometer. The rates of the predicted VC values (\%VC) were calculated as previously reported [14]. Diffusing capacity of the lung for carbon monoxide $(D \mathrm{~L}, \mathrm{CO})$ was measured using a single-breath technique or a re-breathing technique with adjustment to single-breath values. We performed VC in 35 patients and $D$ L,CO in 28 patients among the 37 IPF patients in whom serum levels of periostin were measured.

\section{Establishment of anti-periostin monoclonal and polyclonal antibodies}

We established anti-periostin monoclonal Abs (mAbs) as previously reported [10]. Briefly, 6- to 10-week-old Crj:Wistar rats (Charles River Japan Inc., Kanagawa, Japan) were injected in footpads at least twice with $20 \mu \mathrm{g}$ of recombinant human periostin emulsified in TiterMax Gold adjuvant (TiterMax USA, Norcross, GA, USA). 3 days after the last injection, lymphocytes from popliteal, inguinal and iliac lymph nodes were fused with a Sp2/ $\mathrm{O}$ myeloma cell line. Rat anti-periostin mAbs (immunoglobulin 


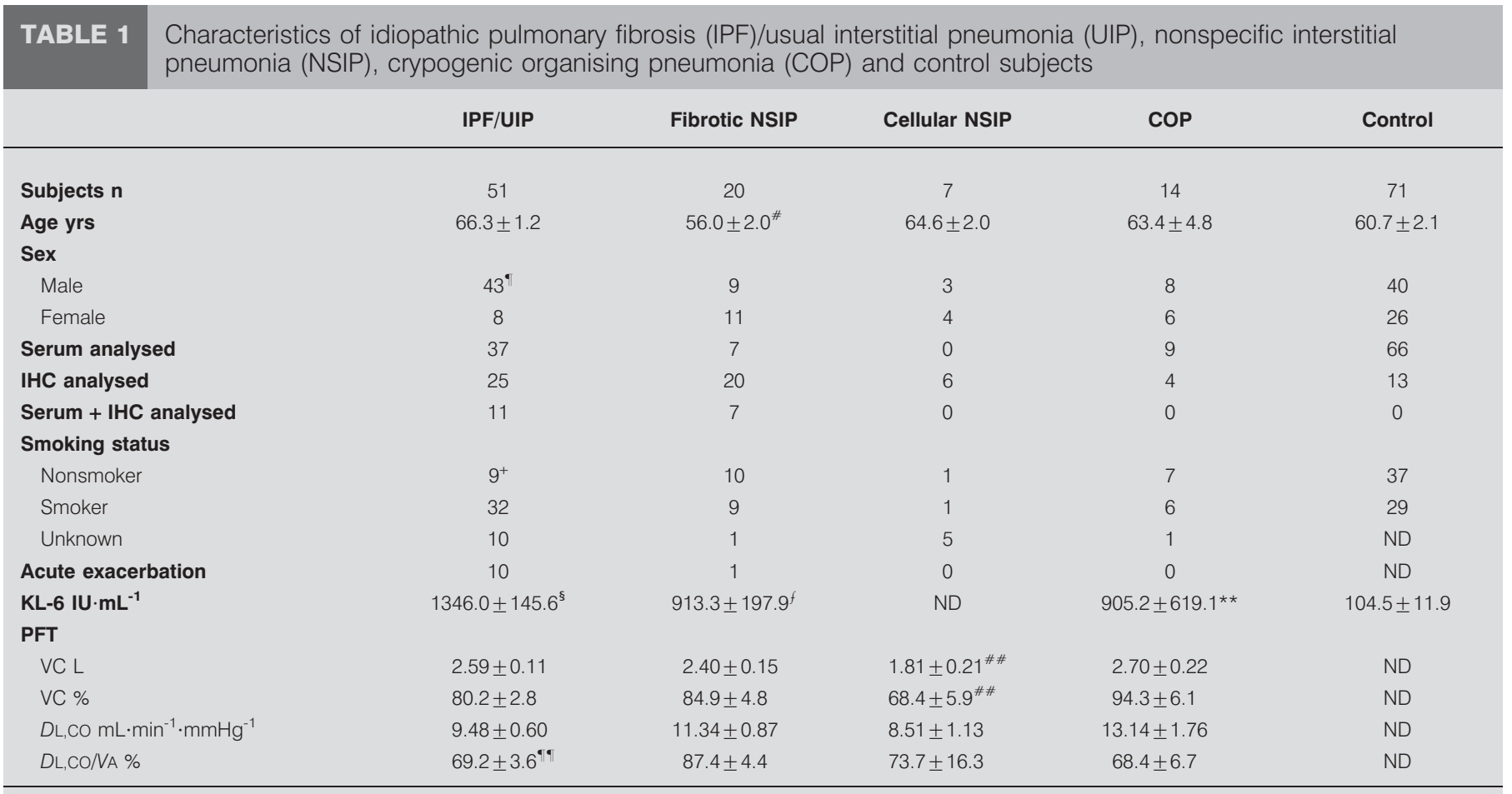

Data are presented as $\mathrm{n}$ or mean \pm SEM, unless otherwise stated. IHC: immunohistochemical; PFT: pulmonary function test; VC: vital capacity; $D \mathrm{~L}, \mathrm{CO}$ : diffusing capacity of the lung for carbon monoxide; VA: alveolar volume; ND: not determined. ${ }^{\#}: p<0.05$ versus IPF/UIP, cellular NSIP and COP; $": p<0.05$ versus fibrotic NSIP, cellular NSIP and control; ${ }^{+}: p<0.05$ versus fibrotic NSIP, COP and control; ${ }^{\xi}: p<0.05$ versus COP and control; ${ }^{f}: p<0.05$ versus control; ${ }^{\# \#}: p<0.05$ versus IPF/UIP, fibrotic NSIP and COP; $"$ : $p<0.05$ versus fibrotic NSIP.

(Ig)G) were purified from culture supernatant of the hybridomas using a protein $G$ affinity chromatography column. Specific pathogen-free rabbits were immunised with recombinant human periostin and antiserum was obtained. Purified rabbit antihuman periostin polyclonal antibody was generated from the antiserum as previously reported [10].

\section{Immunohistochemical assay and immunoreactivity score}

Immunohistochemical analysis was performed as reported previously [15-17]. Briefly, rat anti-human periostin mAbs (clone no. SS19B or SS5D) were used at $4^{\circ} \mathrm{C}$ for $18 \mathrm{~h}$. Positive reactivity was identified using biotin-labelled goat-anti-rat IgG, streptavidin-conjugated peroxidase and 3-3'-diaminobenzidine$4 \mathrm{HCl}$ employing an LSAB2 kit (Dako, Kyoto, Japan). Semiquantitative analysis of the immunohistochemical assay was performed using a modified method that as previously reported [15]. Briefly, we selected specific areas where fibroblasts, intraalveolar macrophages or interstitial mononuclear cell infiltrate appeared to be most markedly observed. In each section, we selected three individual square fields $(0.34 \times 0.27 \mathrm{~mm})$ at $200 \times$. Periostin immunoreactivity scores for each histological feature were defined by counting the numbers of positively stained cells in these areas at $200 \times$ magnification. Two independent examiners evaluated these sections without prior knowledge of the patients' clinical status.

\section{Measurements of periostin by ELISA}

Serum and BALF were obtained from subjects and then stored at $-80^{\circ} \mathrm{C}$ until ELISA assay. We established a human periostin
ELISA assay. Two rat anti-human periostin mAbs (clone no. SS18A and SS17B) were used to establish a sandwich ELISA assay. The SS18A mAb $\left(2 \mu \mathrm{g} \cdot \mathrm{mL}^{-1}\right)$ was incubated overnight at $25^{\circ} \mathrm{C}$ on ELISA plates (loose MaxiSorp ${ }_{\circledR}$ Nunc-Immuno ${ }$ Modules; Thermo Fisher Scientific, Rochester, NY, USA). The ELISA plates were blocked by blocking buffer $(0.5 \%$ casein in TBS, $\mathrm{pH} 8.0$ ) overnight at $4^{\circ} \mathrm{C}$ and then washed three times with washing buffer $(0.05 \%$ Tween 20 in PBS). The ELISA plates were incubated with 100 - to 200 -diluted samples or recombinant periostin standards for $18 \mathrm{~h}$ at $25^{\circ} \mathrm{C}$, followed by washing five times. The peroxidase labelled SS17B $\mathrm{mAb}\left(50 \mathrm{ng} \cdot \mathrm{mL}^{-1}\right)$ was added followed by incubation for $90 \mathrm{~min}$ at $25^{\circ} \mathrm{C}$. After the ELISA plates had been washed five times, reaction solution (0.8 mM 3,3',5,5'-Tetramethylbenzidine, $2.5 \mathrm{mM} \mathrm{H}_{2} \mathrm{O}_{2}$ ) was added, followed by incubation for $10 \mathrm{~min}$ at $25^{\circ} \mathrm{C}$ and then the reaction was stopped by adding the stop solution $(0.7 \mathrm{~N} \mathrm{HCl})$. The values were calculated by subtracting the absorbance at $550 \mathrm{~nm}$ (secondary wavelength) from the absorbance at $450 \mathrm{~nm}$ (primary wavelength) measured by a microplate reader (Bio Rad Laboratories, Tokyo, Japan). Periostin concentrations in the serum were calculated simultaneously using the recombinant periostin proteins. We performed the ELISA assay on duplicated samples.

\section{Statistical analysis}

Data are presented as mean \pm SEM. Differences between the two groups were analysed by the Wilcoxon rank-sum or Fisher's exact test. Correlations between the two groups were evaluated by Spearman's rank correlation coefficient. Survival 
curves were obtained using the Kaplan-Meier method and the difference in survival rates between subgroups was analysed using the log-rank test. $\mathrm{p}<0.05$ was taken to represent statistical significance. The sensitivity and specificity of serum periostin levels for detecting IPF were analysed by using a receiver operating characteristic (ROC) curve generated by logistic regression as previously reported [18]. Statistical analysis was performed with JMP 7.0.1 (SAS Institute Japan, Tokyo, Japan).

\section{RESULTS \\ Clinical findings of IPF, NSIP and COP patients subjected to this study}

Table 1 summarises all subjects studied. It is of note that eight out of 51 IPF patients later showed episodes of acute exacerbation. VC was significantly lower in cNSIP patients than in IPF, fNSIP or COP patients. The mean serum levels of KL-6 greatly and significantly $(p<0.01)$ increased in IPF $(n=37$; $\left.1,346.0 \pm 145.6 \mathrm{U} \cdot \mathrm{mL}^{-1}\right)$, fNSIP $\left(\mathrm{n}=7 ; 913.3 \pm 197.9 \mathrm{U} \cdot \mathrm{mL}^{-1}\right)$ and COP $\left(n=9 ; 905.2 \pm 619.1 \mathrm{U} \cdot \mathrm{mL}^{-1}\right)$ patients compared to the healthy control subjects $\left(n=66 ; 104.5 \pm 11.9 \mathrm{U} \cdot \mathrm{mL}^{-1}\right)$. The levels in IPF and fNSIP patients were slightly higher that those of COP patients $(\mathrm{p}<0.01$ and $\mathrm{p}<0.05$, respectively).

\section{Periostin is a novel component of fibrosis predominant in UIP and INSIP but not in CNSIP and COP}

First, we evaluated 14 rat anti-periostin mAbs to determine whether these mAbs could be used for immunohistochemical analysis. We found that two anti-human periostin mAbs (clones SS19B and SS5D) can be used for the immunohistochemical analysis. Moreover, the positive reactivity (periostin proteins) in lungs of UIP patients detected by these two mAbs were exactly the same as those detected by rabbit anti-human periostin polyclonal antibodies (data not shown). Therefore, in this study we used SS19B and SS5D to perform immunohistochemical analysis.

We investigated the expression of periostin in lung tissues obtained from $25 \mathrm{UIP}, 20 \mathrm{fNSIP}$, seven cNSIP and five COP patients. Normal lung tissues were obtained from five nonsmokers who underwent lung cancer extirpation. Representative periostin expressions in the lung tissues obtained from a representative control subject (69-yr-old female), a UIP patient (64-yr-old male), an fNSIP patient (62-yr-old female), a cNSIP (56-yr-old female) and a COP patient (76-yr-old female) are shown in figures 1 and 2.

Periostin was not observed in pulmonary cells including epithelial cells, alveolar macrophages and most bronchiolar basement membrane of normal lung tissues, but was very weakly deposited in part of the bronchiolar basement membrane (data not shown). In contrast, the lung tissues of UIP showed strong expression of periostin in fibroblasts, especially in FF areas, but not in regenerative alveolar epithelium or macrophages, areas showing established fibrosis or inflammatory cells (fig. 2a). Moreover, in UIP priostin was strongly expressed in the interstitium of alveoli, which appeared to be normal (fig. 2b). In fNSIP, periostin was also strongly expressed in fibroblasts, but not strongly expressed in regenerative alveolar epithelium and macrophages, or inflammatory cells. In cNSIP and COP, periostin was scarcely observed in alveolar septa and inflammatory cells. However, periostin was expressed in the intra-luminal fibrosis of alveolar ducts in COP patients. It has been well recognised that the intra-luminal fibrosis of alveolar ducts in COP patients is potentially reversible. These results demonstrate that periostin is a novel component for ongoing fibroproliferation especially in UIP, fNSIP and COP patients. Semi-quantitative analysis showed that periostin expressing cells in the lungs of UIP $(n=25)$, fNSIP $(n=20)$, cNSIP $(n=6)$ and COP $(n=4)$ were significantly $(\mathrm{p}<0.05)$ increased when compared to normal lung tissues $(n=15)$. In addition, periostin expressing cells in the lungs of UIP were significantly increased as compared to fNSIP, cNSIP and COP (fig. 3).

\section{Serum periostin levels are significantly elevated in IPF patients reflecting expression of periostin in lung tissues}

We then examined whether serum levels of periostin are elevated in IIP patients, reflecting up-regulated expression of periostin in lung tissues. We established a sandwich ELISA assay to detect periostin using two rat anti-human periostin mAbs (clone no. SS18A and SS17B). We confirmed that the limiting level of periostin for detection in this ELISA system is $100 \mathrm{pg} \cdot \mathrm{mL}^{-1}$ (data not shown). We applied serum samples from patients with IPF, fNSIP and COP and from control subjects but we were not able to obtain serum samples from cNSIP patients (fig. 4). Serum periostin levels in IPF patients were significantly elevated $\left(\mathrm{n}=37 ; 107.1 \pm 11.9 \mathrm{ng} \cdot \mathrm{mL}^{-1}\right) \mathrm{com}-$ pared to those in COP patients $\left(\mathrm{n}=9 ; 58.9 \pm 8.2 \mathrm{ng} \cdot \mathrm{mL}^{-1}\right)$ and control subjects $\left(\mathrm{n}=66 ; 39.1 \pm 3.0 \mathrm{ng} \cdot \mathrm{mL}^{-1} ;\right.$ COP patients $p<0.01$, control subjects $p<0.0001$ ). Serum periostin levels of fNSIP were moderate $\left(n=7 ; 77.9 \pm 15.7 \mathrm{ng} \cdot \mathrm{mL}^{-1}\right)$ and statistically different to control subjects $(p<0.01)$ but not to COP patients. In contrast, serum periostin levels in COP patients were only slightly elevated compared to control subjects $(p<0.05)$. Furthermore, periostin in the BALF was also detectable by ELISA in five out of $11 \mathrm{IPF}$ patients (data not shown). Sex, smoking status and KL-6 levels were not associated with serum periostin levels (data not shown). These results show that serum periostin levels are elevated significantly in IPF patients, moderately in fNSIP patients and only slightly in COP patients compared to healthy control subjects, reflecting the expression of periostin in their lung tissues.

\section{IPF is distinguished from control subjects or COP patients by measurement of serum periostin levels with high specificities}

Because serum periostin levels in IPF patients were significantly elevated compared to control subjects and COP patients, we then evaluated the sensitivities and specificities to distinguish IPF from the control or COP by serum periostin levels using a ROC curve (fig. 5). The area under the ROC curve between IPF patients and control subjects was 0.905 . The ROC curve analysis showed that serum periostin levels, with an optimal cut-off point of $95 \mathrm{ng} \cdot \mathrm{mL}^{-1}$, had a good specificity (0.985) and a fair sensitivity (0.514). 19 (51.4\%) out of 37 IPF patients and one $(1.5 \%)$ out of 66 healthy subjects were over the cut-off value. This cut-off value also gave a good specificity (1.0) and a fair sensitivity (0.514) in the comparison study of IPF and COP patients. None of COP patients showed more than the cut-off point of serum periostin level. Furthermore, this cut-off value showed relatively less sensitivity (0.286) in 

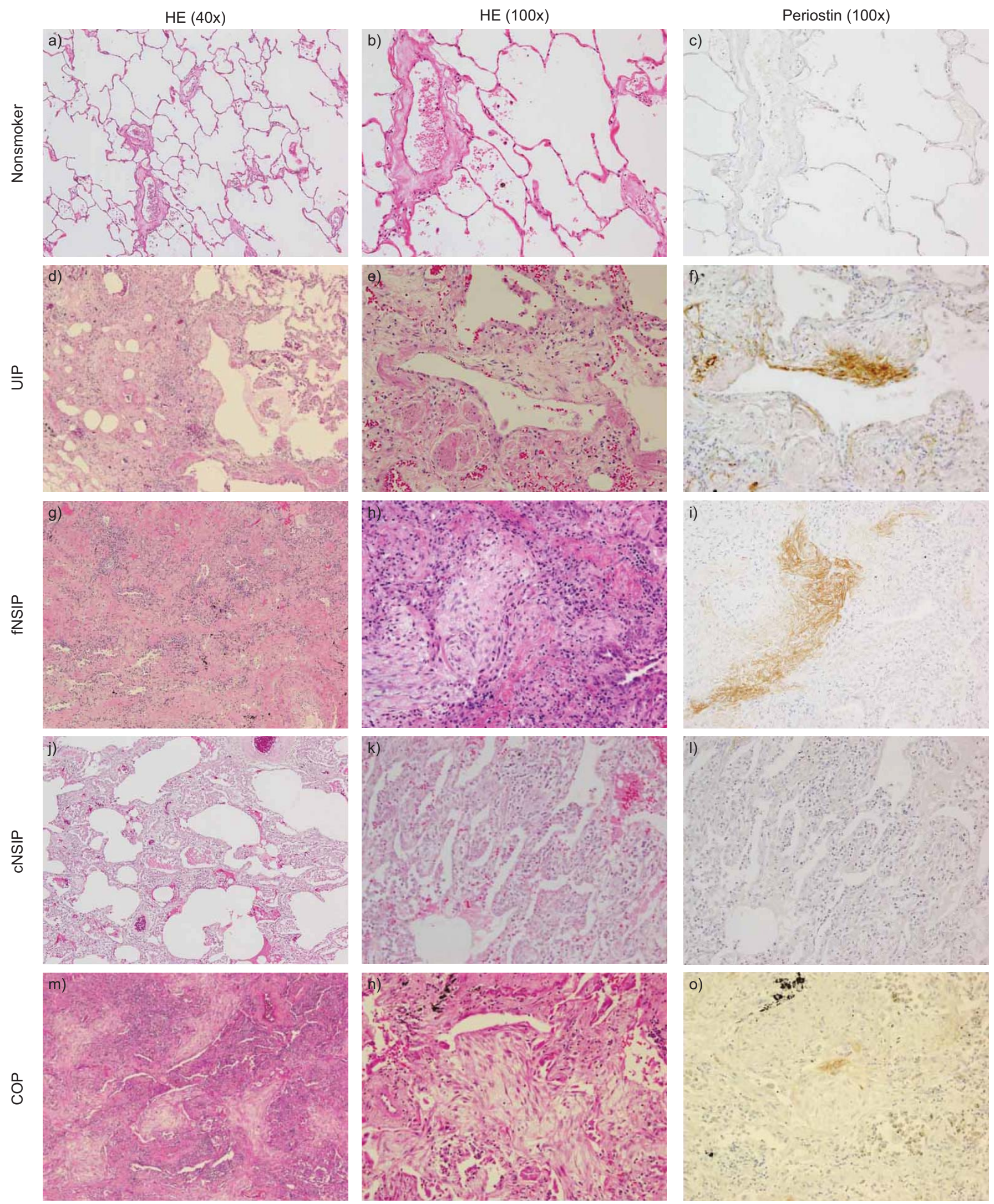

FIGURE 1. Expression of periostin in lungs of a-c) a 69-yr-old female nonsmoker, $d-f$ ) a 64-yr-old male with usual interstitial pneumonia (UIP), g-i) a 62-yr-old female with fibrotic nonspecific interstitial pneumonia (fNSIP), j-I) a 56-yr-old female with cellular nonspecific interstitial pneumonia (cNSIP) and m-o) a 76-yr-old female with crypogenic organising pneumonia (COP). The tissues were stained with haematoxylin and eosin (HE) $40 \times, \mathrm{HE} 100 \times$ or periostin $100 \times$, as shown. 

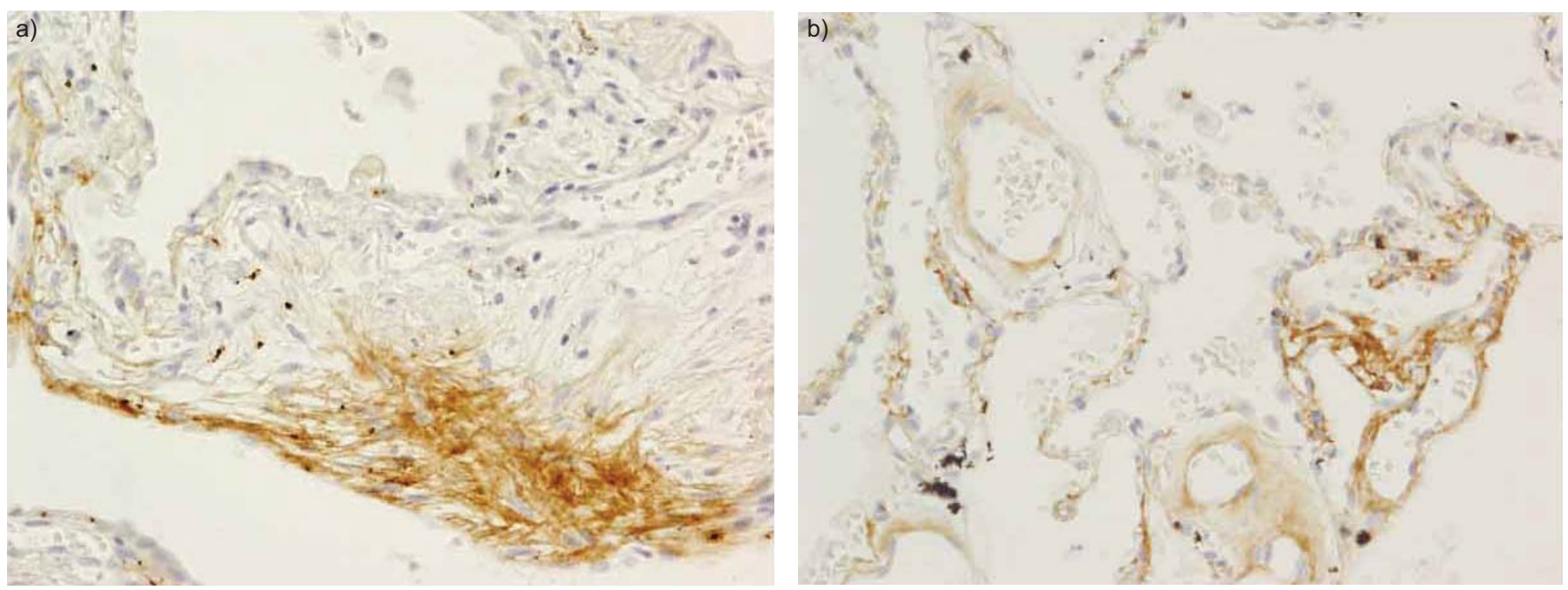

FIGURE 2. Immunohistochemical analysis of periostin in a 64-yr-old male with usual interstitial pneumonia. a) The lung tissues of usual interstitial pneumonia showed strong expression of periostin in fibroblasts, especially in fibrotic foci areas, but not in regenerative alveolar epithelium or macrophages, areas showing established fibrosis or inflammatory cells. b) Moreover, periostin was strongly expressed in the interstitium of alveoli, which seemed to be normal. Magnification at $200 \times$.

the comparison study of fNSIP and healthy subjects. These results demonstrate that IPF can be distinguished from the control or COP by measurement of serum periostin levels using the ELISA system that we established with high specificities.

\section{Serum periostin levels are inversely correlated with the pulmonary functions in IPF patients}

Serum periostin levels in IPF patients were valuable within or above the normal range, indicating the heterogeneous backgrounds of IPF patients. Therefore, we then examined the association between serum periostin levels and various clinical parameters in IPF/UIP patients. There was no correlation between serum periostin levels and sex, smoking status, overall survival rate, BALF cell counts or serum KL-6 levels

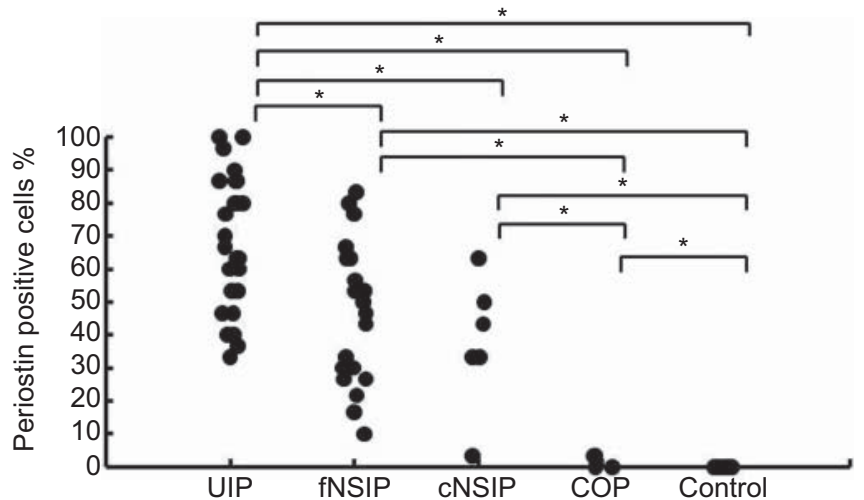

FIGURE 3. Semi-quantitative analysis of immunohistochemical analysis of the lungs of usual interstitial pneumonia (UIP; $n=25$ ), fibrotic nonspecific interstitial pneumonia (fNSIP; $n=20$ ), cellular nonspecific interstitial pneumonia ( $C N S I P ; n=6$ ), crypogenic organising pneumonia (COP; $n=4$ ) patients and control subjects $(n=13)$. Semi-quantitative analysis of the immunohistochemical assay was performed as described in the Methods section. Periostin immunoreactivity scores for each histological feature were defined by counting the numbers of positively stained cells in these areas at $200 \times$ magnification. ${ }^{*}: p<0.05$. (data not shown). In contrast, the 6-month changes in VC $(\mathrm{n}=26)$ and $D \mathrm{~L}, \mathrm{CO}(\mathrm{n}=21)$ showed significant negative correlations with serum periostin levels $(\triangle \mathrm{VC}$ : Spearman $\mathrm{r}=-0.498$, $\mathrm{p}<0.01 ; \Delta D \mathrm{~L}, \mathrm{CO}$ : Spearman $\mathrm{r}=-0.467, \mathrm{p}<0.05$ ) (fig. 6). These results show that serum periostin levels are inversely correlated with the pulmonary functions in IPF patients. In this study, there was no significant correlation between serum KL-6 levels and pulmonary function tests (initial levels and 6-month changes in $\mathrm{VC}$ and $\mathrm{DL}, \mathrm{CO})$. There was no significant differentiation of serum periostin levels between IPF patients with $\left(\mathrm{n}=8,112.9 \pm 35.0 \mathrm{ng} \cdot \mathrm{mL}^{-1}\right)$ and without $(\mathrm{n}=29$,

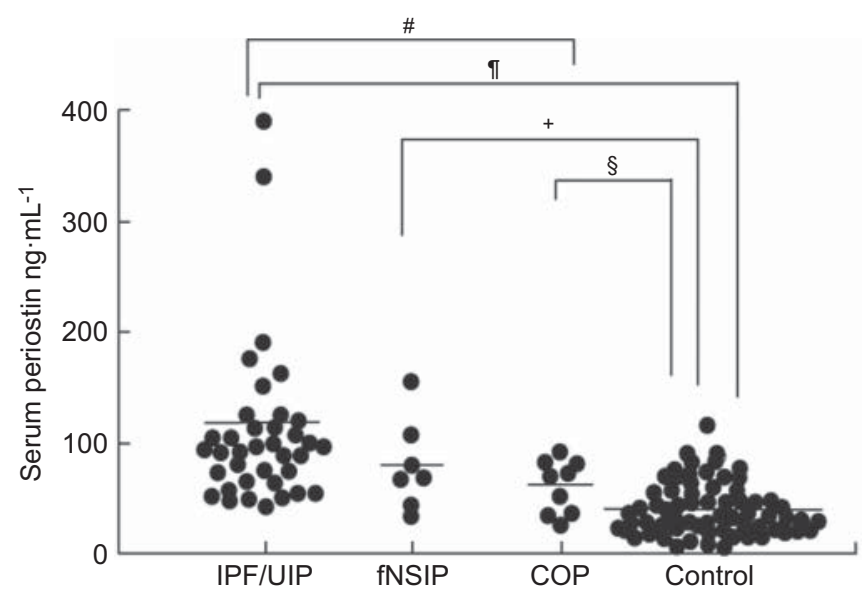

FIGURE 4. Serum periostin levels in sera obtained from idiopathic pulmonary fibrosis (IPF)/usual interstitial pneumonia (UIP; $n=37$ ), fibrotic nonspecific interstitial pneumonia (fNSIP; $n=7$ ), crypogenic organising pneumonia (COP; $n=9$ ) patients and in healthy control subjects $(n=66)$ using an ELISA. All IPF patients were diagnosed by at least two respirologists and at least two radiologists in accordance with the clinical criteria established by the American Thoracic Society/European Respiratory Society [4]. All seven fNSIP and nine COP patients were histopathologically diagnosed by surgical lung biopsy or transbronchial lung biopsy. ${ }^{\#}: p=0.0072 ;{ }^{\bullet}: p<0.0001 ;^{+}: p=0.008 ;^{\varsigma}$ : $p=0.0239$ 


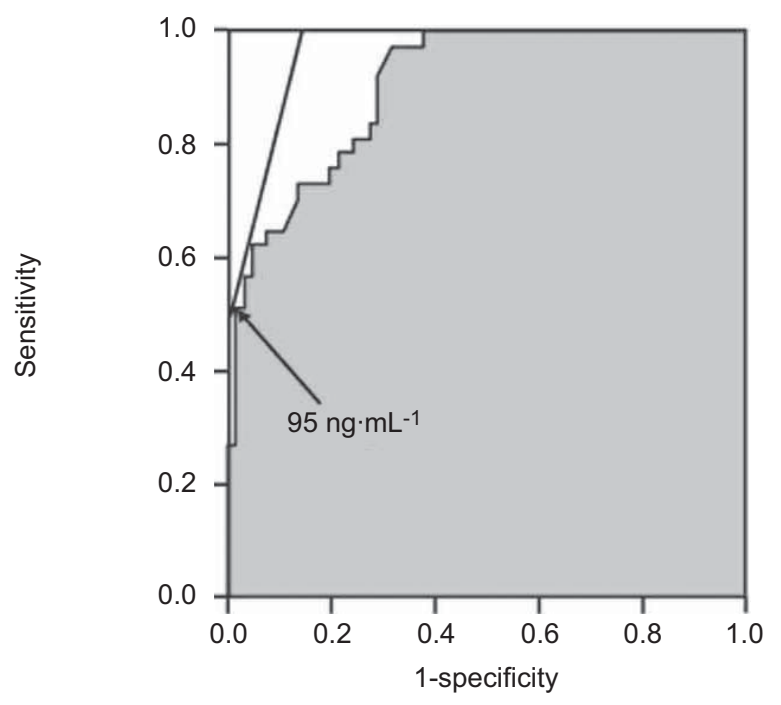

FIGURE 5. Specificity and sensitivity of measurement of serum periostin levels. The receiver operating characteristic (ROC) curve compares serum periostin levels in idiopathic pulmonary fibrosis (IPF) patients and healthy control subjects. The area under the ROC curve between IPF patients and control subjects was 0.905 . The optimal cut-off value of the serum periostin level $\left(95 \mathrm{ng} \cdot \mathrm{mL}^{-1}\right)$ is inserted. The numbers of IPF, fibrotic nonspecific interstitial pneumonia, crypogenic organising pneumonia patients and healthy control subjects over the optimal cutoff value (number over $95 \mathrm{ng} \cdot \mathrm{mL}^{-1}$ ) are: 19/37 (51.4\%), 2/7 (28.6\%), 0/9 (0\%) and $1 / 66(1.5 \%)$, respectively.

$\left.105.6 \pm 12.2 \mathrm{ng} \cdot \mathrm{mL}^{-1}\right)$ acute exacerbation $(\mathrm{p}=0.4948)$. Periostin was not associated with an increased risk of exacerbation in eight IPF patients.

\section{DISCUSSION}

The importance of diagnosing the histopathological types of IIPs has been established as a basis for the prediction of prognoses and responses to treatment. This is due to the fact that IPF/UIP has a poorer prognosis and resists corticosteroid treatment, whereas cNSIP and COP have better prognoses and respond well to such treatment [1-4]. IIP is diagnosed based on the combined clinical, radiological and pathological data of the patients, among which the lung biopsy is the most definitive marker of disease [2-4]. However, the lung biopsy carries a risk of death, with mortality rates ranging from $1 \%$ to $6 \%[19$, 20]. Therefore, it would be of great value if a biomarker to assess histopathological types of IIPs could be identified. In this study we demonstrated that measurement of serum levels of periostin may serve as an important biomarker for characterisation and classification of this condition. ROC curve analysis rendered an optimal cut-off point of $95 \mathrm{ng} \cdot \mathrm{mL}^{-1}$ for the serum periostin level using our ELISA system. Only one $(1.5 \%)$ out of 66 healthy subjects was above the cut-off value, providing good specificity for this assay (0.985). In contrast, 19 $(51.4 \%)$ out of 37 IPF patients and none of the COP patients showed values $>95 \mathrm{ng} \cdot \mathrm{mL}^{-1}$. Our small-scale survey for fNSIP patients showed that two $(28.6 \%)$ out of seven fNSIP patients were above the cut-off value, providing less sensitivity (0.286) than that of IPF. Based on these collective results, we propose that measurement of serum periostin levels is useful to distinguish IPF/UIP from COP or normal lung function. It has been reported that IPF/UIP and fNSIP share a similar
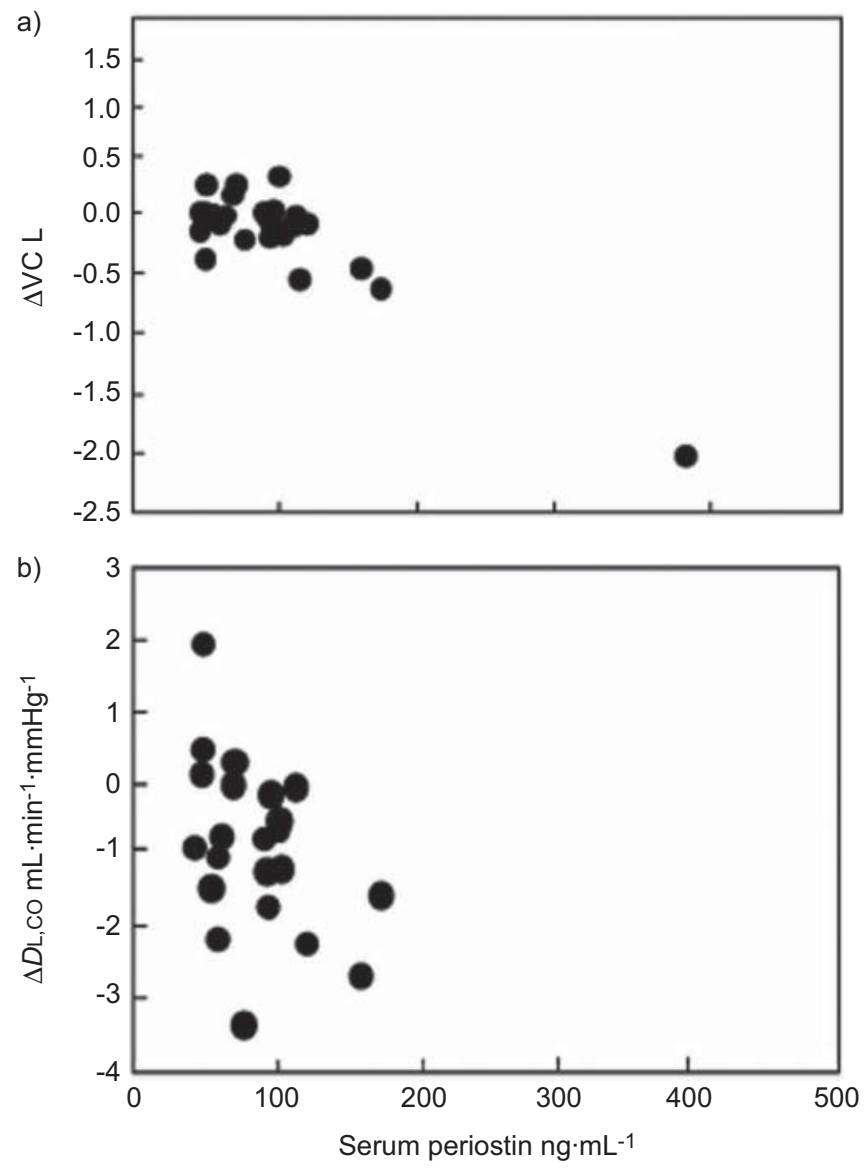

FIGURE 6. Correlation between serum periostin levels and pulmonary functions in idiopathic pulmonary fibrosis (IPF) patients. The correlation between serum periostin levels and 6-month changes in a) vital capacity (VC; $n=26$, $p=0.096, r=0.498)$ and $b$ ) diffusing capacity of the lung for carbon monoxide $(D L, C O ; n=21, p=0.33, r=0.467)$ in IPF patients is shown.

presentation with respect to clinical/radiological/pathological parameters; however, the prognosis of fNSIP is much better than IPF/UIP [1-6]. Semi-quantitative immunohistochemical analysis showed that periostin expressing cells (mainly fibroblasts) in the lungs of UIP were significantly increased as compared to fNSIP. Although there was no a significant difference between IPF and fNSIP, the mean levels of serum periostin in IPF patients were higher $(n=37,107.1 \pm$ $\left.11.9 \mathrm{ng} \cdot \mathrm{mL}^{-1}\right)$ than observed in fNSIP patients $(\mathrm{n}=7,77.9 \pm$ $15.7 \mathrm{ng} \cdot \mathrm{mL}^{-1}$ ). Moreover, $51.4 \%$ (19 out of 37) of IPF and $28.6 \%$ (two out of seven) of fNSIP patients were above the cut-off value $95 \mathrm{ng} \cdot \mathrm{mL}^{-1}$. Therefore, further analysis is needed to determine whether measurement of serum periostin levels will serve as a highly sensitive marker to distinguish IPF/UIP from fNSIP. In addition, we performed preliminary analysis of serum levels of periostin in patients with interstitial lung disease associated with rheumatoid arthritis (RA-ILD). The serum levels of periostin in RA-ILD patients were significantly increased when compared with control subjects (data not shown). Thus, periostin may have an important role in secondary interstitial pneumonia, such as collagen tissue disease-associated ILD. This hypothesis is currently under investigation. 
It has been demonstrated that several biomarkers, such as KL-6, surfactant protein (SP)-A and SP-D, are elevated in serum of IIP patients, thus providing potential biomarkers for the diagnosis of IIPs [21-23]. Particularly, KL-6 has been shown to increase in all of the major types of IIPs including IPF, NSIP and COP. In agreement with these reports, the serum levels of KL-6 were elevated in all of the IPF, fNSIP and COP groups, although, in our study, the levels of COP were slightly less than those of IPF and fNSIP. Periostin is distinct from KL-6 in that IPF/UIP can be distinguished from COP by measurement of serum levels of periostin but not KL-6. Furthermore, we did not observe a correlation between serum levels of periostin and KL-6. These results may be due to the different mechanisms controlling expression of KL-6 and periostin. KL-6, a high molecular glycoprotein (also called mucin 1), is produced by regenerating type II pneumocytes, and is released from the cell surface by shedding [21, 24], whereas periostin is secreted by activated fibroblasts [10]. Thus, periostin may directly reflect lung fibrotic changes in IIPs more than KL-6. It is assumed that combining measurements of periostin with other biomarkers, including KL-6, could further improve the accuracy of diagnosing IIPs.

We analysed serum levels of periostin and various clinical parameters in IPF patients based on the observation that the serum levels of periostin in IPF patients are variable, ranging within the normal parameters to very high values. Consequently, we found that serum levels of periostin in IPF patients correlate with 6-month changes in VC and DL,CO. It is well known that the change in VC (or FVC) is highly correlated with survival rate and that the change in $D \mathrm{~L}, \mathrm{CO}$ has a similar but lesser prognostic value $[3,25,26]$. For example, patients who decline $>10 \%$ of FVC show a poor prognosis with a $5-y r$ survival rate of $20 \%$, whereas patients who improve $>10 \%$ of FVC have a 5 -yr survival rate of $65 \%$. The inverse association between the serum levels of periostin and the pulmonary functions in our study indicates the possible application of serum periostin levels as a prognostic parameter, in addition to the assessment of the histopathological types of IIPs. The use of this marker is also supported by the present finding that periostin was strongly expressed in FF areas in the lungs of UIP patients. These areas consist of aggregating myofibroblasts, which play an important role in ongoing regeneration of fibroblasts in UIP and have been reported to be relevant for predicting the prognosis of UIP patients [3]. Further studies are needed that focus on measuring the serum levels of periostin in individual patients and determine if levels correlate with prognoses.

It is known that several ECM proteins, including type I, III, IV collagens, fibronectin and tenascin- $C$, are involved in the process of fibrosis in UIP patients [5, 6]. Herein, we demonstrated that periostin is a novel component of fibrosis in UIP and fNSIP which negatively correlates with the pulmonary functions in IPF/UIP. To date, the exact role of periostin in the pathogenesis of these diseases remains unknown. It has been previously demonstrated that periostin can interact with other ECM proteins including collagen, tenascin $C$ and fibronectin, and plays an important role in collagen fibrillogenesis or organisation of an extracellular meshwork architecture $[10,27,28]$. Accordingly, a genetic defect of periostin causes impaired amelogenesis in teeth [29], valve abnormalities in the heart [30], and a propensity for cardiac rupture in the healing stage of myocardial infarction [7]. These results increase the possibility that periostin participates in the formation of fibrotic structure in IIPs. As expected, we have found that administration of bleomycin induces less pulmonary fibrotic changes in periostin-deficient mice (M. Uchida; personal communication). These results suggest the possibility that periostin plays an important role in generation of fibrosis in IIPs and that periostin can be a therapeutic target for IIPs.

We found that serum levels of periostin were significantly high in IPF patients, moderate in fNSIP patients, slightly elevated in COP patients compared to normal control subjects, and in parallel with expression of periostin in lung tissues. Furthermore, serum periostin levels were inversely correlated with pulmonary functions in IPF/UIP patients. The distribution of periostin in immunohistochemical studies suggests that it is expressed in areas of fibroblast proliferation. Taken together, our results show that periostin is a novel component of fibrosis in IIPs. Thus, periostin may be a potential biomarker to distinguish IIPs with fibrosis.

\section{SUPPORT STATEMENT}

This work was supported by a Grant-in-Aid for Scientific Research (no. 21590977 to T. Hoshino and no. 20591188 to K. Izuhara) from the Ministry of Education, Science, Sports and Culture of Japan, a grant to the Respiratory Failure Research Group from the Ministry of Health, Labor and Welfare in (Japan; to H. Aizawa), by Okamoto Satoshi Memorial Fund for Pulmonary Fibrosis Research (Tokyo, Japan; to T. Hoshino), and by Collaborative Development of Innovative Seeds, Potentially Verification Stage from Japan Science and Technology Agency (K. Izuhara).

\section{STATEMENT OF INTEREST}

None declared.

\section{ACKNOWLEDGEMENTS}

We thank H.A. Young (NCI-Frederick, Frederick, MD, USA) and D. Wylie for editing and critical review of this manuscript. We also thank E. Kuma, C. Ohki and K. Yamaguchi (Kurume University, Kurume, Japan) for their technical assistance.

\section{REFERENCES}

1 Katzenstein AL, Myers JL. Idiopathic pulmonary fibrosis: clinical relevance of pathologic classification. Am J Respir Crit Care Med 1998; 157: 1301-1315.

2 American Thoracic Society/European Respiratory Society International Multidisciplinary Consensus Classification of the Idiopathic Interstitial Pneumonias. Am J Respir Crit Care Med 2002; 165: 277-304.

3 Kim DS, Collard HR, King TE Jr. Classification and natural history of the idiopathic interstitial pneumonias. Proc Am Thorac Soc 2006; 3: 285-292.

4 Travis WD, Hunninghake G, King TE Jr, et al. Idiopathic nonspecific interstitial pneumonia: report of an American Thoracic Society project. Am J Respir Crit Care Med 2008; 177: 1338-1347.

5 Kuhn C 3rd, Boldt J, King TE Jr, et al. An immunohistochemical study of architectural remodeling and connective tissue synthesis in pulmonary fibrosis. Am Rev Respir Dis 1989; 140: 1693-1703.

6 Kaarteenaho-Wiik R, Tani T, Sormunen R, et al. Tenascin immunoreactivity as a prognostic marker in usual interstitial pneumonia. Am J Respir Crit Care Med 1996; 154: 511-518. 
7 Oka T, Xu J, Kaiser RA, et al. Genetic manipulation of periostin expression reveals a role in cardiac hypertrophy and ventricular remodeling. Circ Res 2007; 101: 313-321.

8 Ruan K, Bao S, Ouyang G. The multifaceted role of periostin in tumorigenesis. Cell Mol Life Sci 2009; 66: 2219-2230.

9 Oku E, Kanaji T, Takata Y, et al. Periostin and bone marrow fibrosis. Int J Hematol 2008; 88: 57-63.

10 Takayama G, Arima K, Kanaji T, et al. Periostin: a novel component of subepithelial fibrosis of bronchial asthma downstream of IL-4 and IL-13 signals. J Allergy Clin Immunol 2006; 118: 98-104.

11 Hayashi N, Yoshimoto T, Izuhara $\mathrm{K}$, et al. $\mathrm{T}$ helper 1 cells stimulated with ovalbumin and IL-18 induce airway hyperresponsiveness and lung fibrosis by IFN-gamma and IL-13 production. Proc Natl Acad Sci USA 2007; 104: 14765-14770.

12 Dina R, Sheppard MN. The histological diagnosis of clinically documented cases of cryptogenic organizing pneumonia: diagnostic features in transbronchial biopsies. Histopathology 1993; 23: 541-545.

13 Collard HR, Moore BB, Flaherty KR, et al. Acute exacerbations of idiopathic pulmonary fibrosis. Am J Respir Crit Care Med 2007; 176: 636-643.

14 Gay SE, Kazerooni EA, Toews GB, et al. Idiopathic pulmonary fibrosis: predicting response to therapy and survival. Am J Respir Crit Care Med 1998; 157: 1063-1072.

15 Kitasato Y, Hoshino T, Okamoto M, et al. Enhanced expression of interleukin-18 and its receptor in idiopathic pulmonary fibrosis. Am J Respir Cell Mol Biol 2004; 31: 619-625.

16 Hoshino T, Okamoto M, Sakazaki Y, et al. Role of proinflammatory cytokines IL-18 and IL-1beta in bleomycin-induced lung injury in humans and mice. Am J Respir Cell Mol Biol 2009; 41: 661-670.

17 Imaoka $\mathrm{H}, \mathrm{Hoshino} \mathrm{T}$, Takei $\mathrm{S}$, et al. Interleukin-18 production and pulmonary function in COPD. Eur Respir J 2008; 31: 287-297.

18 Toda R, Hoshino T, Kawayama T, et al. Validation of "lung age" measured by spirometry and handy electronic FEV1/FEV6 meter in pulmonary diseases. Intern Med (Tokyo, Japan) 2009; 48: 513-521.

19 Kramer MR, Berkman N, Mintz B, et al. The role of open lung biopsy in the management and outcome of patients with diffuse lung disease. Ann Thorac Surg 1998; 65: 198-202.
20 Lettieri CJ, Veerappan GR, Helman DL, et al. Outcomes and safety of surgical lung biopsy for interstitial lung disease. Chest 2005; 127: 1600-1605.

21 Kohno N, Awaya Y, Oyama T, et al. KL-6, a mucin-like glycoprotein, in bronchoalveolar lavage fluid from patients with interstitial lung disease. Am Rev Respir Dis 1993; 148: 637-642.

22 Kuroki Y, Tsutahara S, Shijubo N, et al. Elevated levels of lung surfactant protein $\mathrm{A}$ in sera from patients with idiopathic pulmonary fibrosis and pulmonary alveolar proteinosis. Am Rev Respir Dis 1993; 147: 723-729.

23 Honda Y, Kuroki Y, Matsuura E, et al. Pulmonary surfactant protein D in sera and bronchoalveolar lavage fluids. Am J Respir Crit Care Med 1995; 152: 1860-1866.

24 Thathiah A, Blobel CP, Carson DD. Tumor necrosis factor-alpha converting enzyme/ADAM 17 mediates MUC1 shedding. J Biol Chem 2003; 278: 3386-3394.

25 Flaherty KR, Andrei AC, Murray S, et al. Idiopathic pulmonary fibrosis: prognostic value of changes in physiology and sixminute-walk test. Am J Respir Crit Care Med 2006; 174: 803-809.

26 Latsi PI, du Bois RM, Nicholson AG, et al. Fibrotic idiopathic interstitial pneumonia: the prognostic value of longitudinal functional trends. Am J Respir Crit Care Med 2003; 168: 531-537.

27 Norris RA, Damon B, Mironov V, et al. Periostin regulates collagen fibrillogenesis and the biomechanical properties of connective tissues. J Cell Biochem 2007; 101: 695-711.

28 Kii I, Nishiyama T, Li M, et al. Incorporation of tenascin-C into the extracellular matrix by periostin underlies an extracellular meshwork architecture. J Biol Chem, 285: 2028-2039.

29 Rios H, Koushik SV, Wang H, et al. periostin null mice exhibit dwarfism, incisor enamel defects, and an early-onset periodontal disease-like phenotype. Molecular and cellular biology 2005; 25: 11131-11144.

30 Snider P, Hinton RB, Moreno-Rodriguez RA, et al. Periostin is required for maturation and extracellular matrix stabilization of noncardiomyocyte lineages of the heart. Circ Res 2008; 102: $752-760$. 